\title{
OSCILLATION AND COMPARISON THEOREMS FOR NEUTRAL DIFFERENCE EQUATIONS
}

\author{
B.G. ZHANG* AND PENGXIANG YAN
}

Abstract. In this paper we study qualitative properties of solutions of the neutral difference equation

$$
\left\{\begin{array}{l}
\Delta\left(y_{n}-p y_{n-k}\right)+\sum_{i=1}^{m} q_{n}^{i} y_{n-k_{i}}=0 \\
y_{n}=A_{n} \quad \text { for } n=-M, \ldots,-1,0
\end{array}\right.
$$

where $p \geq 1, M=\max \left\{k, k_{1}, \ldots, k_{m}\right\}$, and $k, k_{i}, i=1, \ldots, m$, are nonnegative integers. Riccati techniques are used.

\section{Introduction}

In a number of recent papers [2-10], the oscillation and nonoscillation of solutions of delay difference equations are being extensively investigated. In paticular the oscillation of solutions of the neutral difference equation

$$
\Delta\left(y_{n}-c y_{n-k}\right)+p_{n} y_{n-m}=0
$$

has been investigated in $[3,6,8,9,10]$, where $p_{n}>0, c \in(0,1), \Delta$ denotes the forward difference operator $\Delta y_{n}=y_{n+1}-y_{n}$. Equation (1.1) was considered by Brayron and Willoughby [1] from the numerical analysis point of view.

In this paper we consider the case that $c \geq 1$ in (1.1), or the equations which we will consider are neutral difference equations of the form

$$
\Delta\left(y_{n}-p y_{n-k}\right)+\sum_{i=1}^{m} q_{n}^{i} y_{n-k_{i}}=0, n=0,1,2, \ldots
$$

Received June 30, 1993; revised March 26, 1994

1991 Mathematics Subject Classification. Primary 39D10.

Key words and phrases. Neutral difference equations, oscillation, comparison theorems.

Research was supported by NNSF of China and Fundation of Shandong Province 
Let $M=\max \left\{k, k_{1}, \ldots, k_{m}\right\}, I_{m}=\{1, \ldots, m\}, \tilde{k}=\max _{\left\{i \in I_{m}\right\}} k_{i}$. where $k, k_{i}$ are nonnegative integers $i=1, \ldots, m$ and $p \geq 1$.

By a solution of (1.2) we mean a sequence $\left\{y_{n}\right\}$ which is defined for $n \geq-M$ and satisfies equation (1.2) for $n=0,1,2, \ldots$, clearly, if

$$
y_{n}=A_{n}, \text { for } n=-M, \ldots,-1,0
$$

are given, then equation (1.2) has a unique solution satisfying the initial condition (1.3). A nontrivial solution $\left\{y_{n}\right\}$ of equation (1.2) is said to be oscillatory if for every $N>0$ there exists an $n \geq N$ such that $y_{n} y_{n+1} \leq 0$, otherwise it is nonoscillatory. In this paper sufficient conditions for all solutions of (1.2) to be oscillatory and (1.2) to have a nonoscillatory solution are obtained respectively. As a consequence we prove that the oscillation of equation (1.2) with periodic coefficients is equivalent to the equation with constant coefficients. Finally, a comparison result for the oscillation of equation (1.2) is derived.

\section{Main Results}

We assume through out this paper that $(H) \sum_{i=1}^{m} q_{n}^{i}$ can not be identically zero on $\left[N_{1}, N_{2}\right]$ with $N_{1}<N_{2}$ where $N_{1}, N_{2}$ are any two positive integers

Lemma 2.1. Assume that $p \geq 1, q_{n}^{i} \geq 0, i \in I_{m}$ and

$$
\sum_{j=N}^{\infty} \sum_{i=1}^{m} q_{j}^{i}=\infty
$$

Let $\left\{y_{n}\right\}$ be an eventually positive solution of (1.2). Then $z_{n}<0$ and $\Delta z_{n}<0$ eventually, where

$$
z_{n}=y_{n}-p y_{n-k} .
$$

Proof. From (1.2), $\Delta z_{n}<0$. If $z_{n} \geq 0$, then $y_{n} \geq p y_{n-k}$ which implies that there exists an $d>0$ such that $y_{n} \geq d>0$ for all large $n$. Hence from (1.2)

$$
\Delta z_{n}+d \sum_{i=1}^{m} q_{n}^{i} \leq 0
$$

(2.1) and (2.3) lead to that $z_{n} \rightarrow-\infty$ as $n \rightarrow \infty$, a contradiction. Therefore $z_{n}<0$ eventually. The proof is completed.

Lemma 2.2. In addition to assumptions of Lemma 2.1, we further assume that

$$
q_{n}^{i} \leq q_{n-k}^{i}, \quad i \in I_{m}, n=N, N+1, \ldots
$$


Let $\left\{y_{n}\right\}$ be an eventually positive solution of (1.2) and $z_{n}$ is defined by (2.2). Set

$$
w_{n}=\frac{\Delta z_{n}}{z_{n}}>0
$$

The eventually

$$
w_{n} \geq \frac{1}{p} w_{n+k} \prod_{i=n}^{n+k-1}\left(1+w_{i}\right)+\frac{1}{p} \sum_{i=1}^{m} q_{n}^{i} \prod_{i=n}^{n+k-k_{i}-1}\left(1+w_{i}\right) .
$$

Proof. From (2.1) and (2.2) we have

$$
\begin{aligned}
\Delta z_{n} & =-\sum_{i=1}^{m} q_{n}^{i} y_{n-k_{i}} \\
& =-\sum_{i=1}^{m} q_{n}^{i}\left(y_{n-k_{i}}-p y_{n-k_{i}-k}+p y_{n-k_{i}-k}\right) \\
& =-\sum_{i=1}^{m} q_{n}^{i} z_{n-k_{i}}-p \sum_{i=1}^{m} q_{n}^{i} y_{n-k_{i}-k} \\
& \geq-\sum_{i=1}^{m} q_{n}^{i} z_{n-k_{i}}-p \sum_{i=1}^{m} q_{n-k}^{i} y_{n-k_{i}-k} \\
& =-\sum_{i=1}^{m} q_{n}^{i} z_{n-k_{i}}+p \Delta z_{n-k} .
\end{aligned}
$$

Exchanging terms in the above inequality we obtain

$$
\begin{gathered}
\Delta z_{n} \leq \frac{\Delta z_{n+k}}{p}+\frac{1}{p} \sum_{i=1}^{m} q_{n+k}^{i} z_{n+k-k_{i}} \\
\frac{\Delta z_{n}}{z_{n}} \geq \frac{\Delta z_{n+k}}{p z_{n}}+\frac{1}{p z_{n}} \sum_{i=1}^{m} q_{n+k}^{i} z_{n+k-k_{i}} .
\end{gathered}
$$

By (2.5), $z_{n+1} / z_{n}=1+w_{n}$ and hence

$$
\frac{z_{n+k}}{z_{n}}=\left(1+w_{n+k-1}\right) \ldots\left(1+w_{n}\right) .
$$

Substituting (2.5) into (2.7) we obtain (2.6). The proof is finished.

Remark 2.1. If $m=1$ and $q_{n}>0$ for all large $n$, then (2.4) is not necessary. (2.6) is replaced by

$$
w_{n} \geq \frac{q_{n}}{p q_{n+k}} w_{n+k} \prod_{i=n}^{n+k-1}\left(1+w_{i}\right)+\frac{q_{n}}{p} \prod_{i=n}^{n+k-k_{1}-1}\left(1+w_{i}\right) .
$$


Theorem 2.1. Assume that

(1) $p>1, k \geq \tilde{k}+2$;

(2) $q_{n}^{i} \geq 0, n=N-k, N-k+1, \ldots, q_{n}^{i} \leq q_{n-k}^{i}, \mathbf{i} \in I_{m}, n=N, N+1, \ldots$;

$$
\liminf _{n \rightarrow \infty} \sum_{j=n}^{n+\left[\frac{k-\bar{k}}{2}\right]-1}\left(\sum_{i=1}^{m} q_{j}^{i}\right)>0
$$

(3)

$$
\inf _{n \geq N} \min _{\mu>0}\left\{\frac{1}{p}(1+\mu)^{k}+\frac{1}{p \mu} \sum_{i=1}^{m}\left(\frac{1}{T} \sum_{j=n}^{n+k-k_{i}, i \in I_{m}} q_{j+k}^{i}\right)(1+\mu)^{k-k_{i}}\right\}>1 .
$$

Then every solution of (1.2) is oscillatory.

Proof. Suppose the contrary. Let $\left\{y_{n}\right\}$ be a positive solution of (1.2). It is easy to see that (2.10) implies (2.1). Then Lemma 2.2 holds, i.e., (2.6) holds. Define sequence $\left\{\lambda_{n}^{(l)}\right\}, n=N, N+1, \ldots, l=1,2, \ldots$ as follows:

$$
\begin{gathered}
\left\{\lambda_{n}^{(l)}\right\}=\{0\}, n=N, N+1, \ldots, \\
\lambda_{n}^{(l+1)}=\frac{1}{p} \lambda_{n+k}^{(l)} \prod_{i=n}^{n+k-1}\left(1+\lambda_{i}^{(l)}\right)+\frac{1}{p} \sum_{i=1}^{m} q_{n+k}^{i} \prod_{i=n}^{n+k-k_{i}-1}\left(1+\lambda_{i}^{(l)}\right) . \\
n=N, N+1, \ldots, l=1,2, \ldots
\end{gathered}
$$

Define a sequence of numbers as follows:

$$
\begin{gathered}
\mu_{1}=0 \\
\mu_{r+1}=\inf _{n \geq N} \min _{T=k, k-k_{i}, i \in I_{m}}\left[\frac{1}{p} \mu_{r}\left(1+\mu_{r}\right)^{k}+\frac{1}{p} \sum_{i=1}^{m}\left(\frac{1}{T} \sum_{j=n}^{n+T-1} q_{j+k}^{i}\right)\left(1+\mu_{r}\right)^{k-k_{i}}\right] \\
r=1,2, \ldots
\end{gathered}
$$

Condition (3) implies that

$$
0=\mu_{1}<\mu_{2}<\ldots
$$

(2.6) implies that

$$
\lambda_{n}^{(l+1)} \leq w_{n}, l=0,1,2, \ldots, n=N, N+1, \ldots
$$

and

$$
\frac{1}{T} \sum_{j=n}^{n+T-1} \lambda_{n}^{(l+1)} \geq \mu_{l+1}, l=0,1,2, \ldots
$$


where $T=k, k-k_{i}, i \in I_{m}, n=N, N+1, \ldots$ From (2.12) and condition (3), if $\mu^{*}=$ $\lim _{k \rightarrow \infty} \mu_{k}$ exists and $\mu^{*}$ is finite, then

$$
\mu^{*}=\inf _{n \geq N} \min _{T=k, k-k_{i}, i \in I_{m}}\left\{\frac{1}{p} \mu^{*}\left(1+\mu^{*}\right)^{k}+\frac{1}{p} \sum_{i=1}^{m}\left(\frac{1}{T} \sum_{j=n}^{n+T-1} q_{j+k}^{i}\right)\left(1+\mu^{*}\right)^{k-k_{i}}\right\}
$$

hence

$$
\inf _{n \geq N, \mu>0} \min _{T=k, k-k_{i}, i \in I_{m}}\left\{\frac{1}{p}(1+\mu)^{k}+\frac{1}{p \mu} \sum_{i=1}^{m}\left(\frac{1}{T} \sum_{j=n}^{n+T-1} q_{j+k}^{i}\right)(1+\mu)^{k-k_{i}}\right\} \leq 1,
$$

a contradiction. Therefore $\lim _{k \rightarrow \infty} \mu_{k}=+\infty$. Hence from (2.12) and (2.13) we have

$$
\frac{1}{T} \sum_{j=n}^{n+T-1} w_{j} \rightarrow \infty \text { as } n \rightarrow \infty
$$

and hence from (2.8)

$$
\frac{z_{n+T}}{z_{n}} \rightarrow \infty \text { as } n \rightarrow \infty .
$$

On the other hand, $z_{n}>-p y_{n-k}$, therefore from Lemma 2.1

$$
\Delta z_{n}=-\sum_{i=1}^{m} q_{n}^{i} y_{n-k_{i}}<\frac{1}{p} \sum_{i=1}^{m} q_{n}^{i} z_{n+k-k_{i}}<\left(\frac{1}{p} \sum_{i=1}^{m} q_{n}^{i}\right) z_{n+k-\bar{k}}
$$

Hence

$$
\sum_{j=n}^{n+\left[\frac{k-\bar{k}}{2}\right]-1}\left(\frac{1}{p} \sum_{i=1}^{m} q_{j}^{i}\right) \frac{z_{n+k-\bar{k}}}{z_{n+\left[\frac{k-\bar{k}}{2}\right]}} \leq 1 .
$$

By (2.10) and (2.15) we have

$$
z_{n+k-\tilde{k}} / z_{n+\left[\frac{k-\bar{k}}{2}\right]} \text { is bounded, }
$$

which contradicts (2.14). The proof is completed.

For $m=1$, from (2.9) the following theorem holds.

Theorem 2.2. Assume that

(1) $p>1, k \geq k_{1}+2$;

(2) $q_{n}>0$ and

$$
\liminf _{n \rightarrow \infty} \sum_{j=n}^{n+\left[\frac{k-k_{1}}{2}\right]-1} q_{j}>0
$$


(3)

$$
\operatorname{infmin}_{n \geq N, \mu>0}\left\{\frac{(1+\mu)^{k}}{p\left(k-k_{1}\right)} \sum_{i=n}^{n+k-k_{1}-1} \frac{q_{i}}{q_{i+k}}+\frac{(1+\mu)^{k-k_{1}}}{p \mu\left(k-k_{1}\right)} \sum_{i=n}^{n+k-k_{1}-1} q_{i}\right\}>1 .
$$

Then every solution of (1.2) is oscillatory.

Theorem 2.3. Assume that

(1) $p>1, k>k_{i}, i \in I_{m}$;

(2)

$$
\liminf _{n \rightarrow \infty}\left(\sum_{i=1}^{m} q_{n}^{i}\right)>0
$$

(3) there exist $\mu>0$ and $N$ such that

$$
\sup _{n \geq N, T=k, k-k_{i}, i \in I_{m}}\left[\frac{1}{p}(1+\mu)^{k}+\frac{1}{p \mu} \sum_{i=1}^{m} \frac{1}{T}\left(\sum_{j=n}^{n+T-1} q_{j+k}^{i}\right)(1+\mu)^{k-k_{i}}\right] \leq 1 .
$$

Then equation (1.2) has a positive solution.

Proof. Define

$$
\begin{gathered}
\left\{\lambda_{n}^{(l)}\right\}=\{0\}, n=N, N+1, \ldots \\
\lambda_{n}^{(l+1)}=\frac{1}{p} \lambda_{n+k}^{(l)} \prod_{i=n}^{n+k-1}\left(1+\lambda_{i}^{(l)}\right)+\frac{1}{p} \sum_{i=1}^{m} q_{n+k}^{i} \prod_{i=n}^{n+k-k_{i}-1}\left(1+\lambda_{i}^{(l)}\right) \\
n=N, N+1, \ldots, l=1,2, \ldots
\end{gathered}
$$

Clearly $\lambda_{n}^{(l)} \leq \lambda_{n}^{(l+1)}, l=1,2, \ldots, n=N, N+1, \ldots$. We claim that

$$
\frac{1}{T} \sum_{i=n}^{n+T-1} \lambda_{i}^{(l)} \leq \mu, n \geq N
$$

In fact, $l=1$ is true. Assume that (2.18) is true for some $l^{\prime}$, then

$$
\begin{aligned}
\frac{1}{T} \sum_{i=n}^{n+T-1} \lambda_{i}^{\left(l^{\prime}+1\right)} & =\frac{1}{T} \sum_{i=n}^{n+T-1}\left\{\frac{1}{p} \lambda_{i+k}^{\left(l^{\prime}\right)} \prod_{j=i}^{i+k-1}\left(1+\lambda_{j}^{\left(l^{\prime}\right)}\right)+\frac{1}{p} \sum_{j=1}^{m} q_{i+k}^{j} \prod_{j=i}^{i+k-k_{j}-1}\left(1+\lambda_{j}^{\left(l^{\prime}\right)}\right)\right\} \\
& \leq \frac{\mu}{p}(1+\mu)^{k}+\frac{1}{p T} \sum_{i=n}^{n+T-1}\left(\sum_{j=1}^{m} q_{i+k}^{j}\right)(1+\mu)^{k-k_{i}} \leq \mu .
\end{aligned}
$$

Hence $\left\{\lambda_{n}^{(l)}\right\} \rightarrow\left\{\lambda_{n}\right\}$ as $l \rightarrow \infty, n=N, N+1, \ldots$, and

$$
\frac{1}{T} \sum_{i=n}^{n+T-1} \lambda_{i} \leq \mu, n \geq N
$$


and

$$
\lambda_{n}=\frac{1}{p} \lambda_{n+k} \prod_{i=n}^{n+k-1}\left(1+\lambda_{i}\right)+\frac{1}{p} \sum_{i=1}^{m} q_{n+k}^{i} \prod_{i=n}^{n+k-k_{i}-1}\left(1+\lambda_{i}\right), n \geq N .
$$

Set $z_{N}=1, \frac{z_{n+1}}{z_{n}}=1+\lambda_{n}, n=N, N+1, \ldots$, therefore

$$
\frac{z_{n}}{z_{N}}=z_{n}=\prod_{i=N}^{n-1}\left(1+\lambda_{n}\right), \lambda_{n}=\frac{\Delta z_{n}}{z_{n}}
$$

hence (2.19) becomes

$$
\frac{\Delta z_{n}}{z_{N}}=\frac{1}{p} \frac{\Delta z_{n+k}}{z_{n+k}} \frac{z_{n+k}}{z_{n}}+\frac{1}{p} \sum_{i=1}^{m} q_{n+k}^{i} \frac{z_{n+k-k_{i}}}{z_{n}}
$$

and hence

$$
\begin{gathered}
\Delta z_{n}=\frac{1}{p} \Delta z_{n+k}+\frac{1}{p} \sum_{i=1}^{m} q_{n+k}^{i} z_{n+k-k_{i}} \\
p \Delta z_{n}=\Delta z_{n+k}+\sum_{i=1}^{m} q_{n+k}^{i} z_{n+k-k_{i}}
\end{gathered}
$$

which implies that equation (1.2) has a positive solution

$$
z_{N}=1, z_{n}=\prod_{i=N}^{n-1}\left(1+\lambda_{i}\right), n=N+1, \ldots
$$

For $m=1$, we have the following

Theorem 2.4. Assume that

(1) $p>1, k>k_{1}$;

(2) $\liminf _{n \rightarrow \infty} q_{n}>0$;

(3) There exist $\mu>0$ and $N$ such that

$$
\sup _{n \geq N}\left\{\frac{q_{n}}{p q_{n+k}}(1+\mu)^{k}+\frac{q_{n}}{p \mu}(1+\mu)^{k-k_{1}}\right\} \leq 1
$$

Then equation (1.2) has a positive solution.

Corollary 2.1. If $m=1, p>1, k>k_{1}, q_{n}=q>0$, then every solution of (1.2) is oscillatory if and only if

$$
\inf _{n \geq N, \mu>0}\left\{\frac{1}{p}(1+\mu)^{k}+\frac{q}{p \mu}(1+\mu)^{k-k_{i}}\right\}>1 .
$$


Remark 2.2. Theorem 2.2 includes Theorem 4.1 of [8] and Theorem 4 (a) in [4], since

$$
\min _{\mu>0} \frac{(1+\mu)^{k-k_{1}}}{\mu}=\frac{\left(k-k_{1}\right)^{k-k_{1}}}{\left(k-k_{1}-1\right)^{k-k_{1}-1}} .
$$

Remark 2.3. Equation (1.2) with constant coefficients has been studied in [3]. The result in Corollary 2.1 is better.

As an application of Theorem 2.1 and 2.3 we consider equation (1.2) with periodic coefficients, i.e., we assume that there exists an integer $\delta>0$ such that

$$
q_{n+\delta}^{i}=q_{n}^{i}, i \in I_{m} \text { for any } n
$$

Then

$$
\frac{1}{\delta} \sum_{j=n}^{n+\delta-1} q_{j}^{i}=\tilde{q}^{i}
$$

is a constant, $i \in I_{m}$.

Theorem 2.5. Assume that $p \geq 1$ and there exist positive integers $m_{0}$ and $m_{i}, i \in I_{m}$ such that $k=m_{0} \delta, k_{i}=m_{i} \delta, k-k_{i} \geq 2, i \in I_{m}$. Then following statements are equivalent.

(1) Every solution of (1.2) is oscillatory;

(2) Every solution of the neutral difference equation with constant coefficients

$$
\Delta\left(y_{n}-p y_{n-k}\right)+\sum_{i=1}^{m} \tilde{q}^{i} y_{n-k_{i}}=0
$$

is oscillatory.

Proof. Suppose that (2.24) has a nonoscillatory solution. Then the characteristic equation [6]

$$
(\lambda-1)\left(1-p \lambda^{-k}\right)+\sum_{i=1}^{m} \tilde{q}^{i} \lambda^{-k_{i}}=0
$$

has a positive root. It is easy to see that $\lambda>1$. Set $\lambda=1+\mu$. Then (2.25) is reduced to

$$
\frac{1}{p}(1+\mu)^{k}+\frac{1}{p \mu} \sum_{i=1}^{m} \tilde{q}^{i}(1+\mu)^{k-k_{i}}=1,
$$

which implies that Theorem 2.3 holds. Hence (1.2) has a positive solution, a contraction. If (2) holds, then (2.25) has no real roots. Therefore

$$
(\lambda-1)\left(1-p \lambda^{-k}\right)+\sum_{i=1}^{m} \tilde{q}^{i} \lambda^{-k_{i}}>0 .
$$


Setting $\lambda=1+\mu$, then we have

$$
\frac{1}{p}(1+\mu)^{k}+\frac{1}{p \mu} \sum_{i=1}^{m} \tilde{q}^{i}(1+\mu)^{k-k_{i}}>1
$$

By Theorem 2.1, every solution of (1.2) is oscillatory. The proof is completed.

Remark 2.4. Using the average method to Theorems 3.5 and 3.6 in [8], it is not difficult to show that Theorem 2.5 is true for $p \in[0,1]$ too, where $k>\tilde{k}$ is not required.

Now we present a comparison result for oscillation of (1.2). we consider (1.2) associated with

$$
\Delta\left(y_{n}-P y_{n-k}\right)+\sum_{i=1}^{m} Q_{n}^{i} y_{n-k_{i}}=0 .
$$

Theorem 2.6. Assume that

(1) $k \geq \tilde{k}+2$;

(2) $P>1, Q_{n-k}^{i} \geq Q_{n}^{i}>0, \sum_{j=N}^{\infty} \sum_{i=1}^{n} Q_{j}^{i}=\infty$ and

$$
\frac{P}{p} \leq \frac{Q_{n}^{i}}{q_{n}^{i}} \leq 1, n=N, N+1, \ldots, i \in I_{m} .
$$

Then if every solution of (1.2) is oscillatory, so is (2.29).

Proof. Suppose not. Iet $\left\{x_{n}\right\}$ be a positive solution of (2.29). By Lemma 2.2,

$$
u_{n} \geq \frac{1}{P} u_{n+k} \prod_{i=n}^{n+k-1}\left(1+u_{i}\right)+\frac{1}{P} \sum_{i=1}^{m} Q_{n+k}^{i} \prod_{i=n}^{n+k-k_{i}-1}\left(1+u_{i}\right)
$$

where

$$
u_{i}=\frac{\Delta\left(x_{i}-p x_{i-k}\right)}{x_{i}-p x_{i-k}}>0
$$

In view of (2)

$$
u_{n} \geq \frac{1}{p} u_{n+k} \prod_{i=n}^{n+k-1}\left(1+u_{i}\right)+\frac{1}{p} \sum_{i=1}^{m} q_{n+k}^{i} \prod_{i=n}^{n+k-k_{i}-1}\left(1+u_{i}\right) .
$$

Define

$$
\left\{\lambda_{n}^{(l)}\right\}=\left\{u_{n}\right\}, n=N, N+1, \ldots
$$

and

$$
\lambda_{n}^{(l+1)}=\frac{1}{p} \lambda_{n+k}^{(l)} \prod_{i=n}^{n+k-1}\left(1+\lambda_{i}^{(l)}\right)+\frac{1}{p} \sum_{i=1}^{m} q_{n+k}^{i} \prod_{i=n}^{n+k-k_{i}-1}\left(1+\lambda_{i}^{(l)}\right) .
$$




$$
n=N, N+1, \ldots, l=1,2, \ldots
$$

In view of (2.31), we have

$$
\lambda_{n}^{(l+1)} \leq \lambda_{n}^{(l)}, l=1,2, \ldots, n=N, N+1, \ldots
$$

Hence

$$
\lim _{l \rightarrow \infty} \lambda_{n}^{(l)}=\lambda_{n}
$$

exists and $\lambda_{n}>0$ satisfies

$$
\lambda_{n}=\frac{1}{p} \lambda_{n+k} \prod_{i=n}^{n+k-1}\left(1+\lambda_{i}\right)+\frac{1}{p} \sum_{i=1}^{m} q_{n+k}^{i} \prod_{i=n}^{n+k-k_{i}-1}\left(1+\lambda_{i}\right) .
$$

Similar to Theorem 2.3, we obtain a positive solution of (1.2)

$$
z_{N}=1, z_{n}=\prod_{i=N}^{n-1}\left(1+\lambda_{i}\right), n=N+1, \ldots
$$

which contradicts the assumption. The proof is completed.

\section{References}

[1] R. K. Brayron and R. A. Willoughby, "On the numerical integration of a symmetric system of difference differential equations of neutral type," J. Math. Anal. Appl., 18 (1969), 182-189.

[2] L. H. Erbe and B. G. Zhang, "Oscillation of discrete analogues of delay equations," Differential and Integral Equations, 2 (1989), N.3, 300-309.

[3] D. A. Gedrgion, E. A. Grove and G. Ladas, "Oscillation of neutral difference equations," Appl. Anal., 33 (1989), N.3-4, 243-253.

[4] - - "Oscillation of neutral difference equation with variable coefficients," Differential Equations, Lecture Notes in Pure and Applied Mathematics, Ed. Saber Elaydi, Marcel Dekker, 127 (1991), 165-173.

[5] I. Gyori and G. Ladas, Oscillation Theory of Delay Differential Equations wiih Applications, Clarenden Press, Oxford, 1991.

[6] G. Ladas, "Recent developments in the oscillation of delay difference equations," Differential Equations: Stability and Control (1990), Marcel Dekker, 321-332.

[7] G. Ladas, C. G. Philos and Y. G. Sficas, "Necessary and sufficient conditions for the oscillation of difference equations," Liberta Math., 9 (1989), 121-125.

[8] B. S. Lalli, B. G. Zhang and Li Juan Zhao, "On oscillation and existence of positive solutions of neutral difference equations," J. Math. Anal. Appl., 158 (1991), N.1, 213-233.

[9] B. S. Lalli, B. G. Zhang, "On existence of positive solutions and bounded oscilation for neutral difference equations," J. Math. Anal. Appl., 166 (1992), N.1, 272-287.

[10] Zhicheng Wang and Jianshe Yu, "Oscillation criteria for second order nonliner difference equations," Funkcial Ekvac, 34 (1991), N.2, 313-319.

Department of Applied Mathematics, Ocean University of Qingdao, Qingdao, China 266003.

Department of Mathematics, University of Alberta, Edmonton, Alberta, Canada T6G 2 G1. 